\title{
Significance of the neutrophil-to-lymphocyte ratio in young patients with oral squamous cell carcinoma
}

This article was published in the following Dove Press journal:

Cancer Management and Research

\author{
Baixia Zhang' \\ Wei $\mathrm{Du}^{2}$ \\ Kang Gan' \\ Qigen Fang ${ }^{2}$ \\ Xu Zhang ${ }^{2}$ \\ 'Department of Stomatology, The First \\ Affiliated Hospital of Zhengzhou \\ University, Zhengzhou, Henan Province, \\ People's Republic of China; ${ }^{2}$ Department \\ of Head Neck and Thyroid, Affiliated \\ Cancer Hospital of Zhengzhou \\ University, Henan Cancer Hospital, \\ Zhengzhou, Henan Province, People's \\ Republic of China
}

Background: The main goal of this study was to evaluate the prognosis of young patients with oral squamous cell carcinoma (SCC) with a focus on the value of the pretreatment neutrophil-to-lymphocyte ratio (NLR).

Materials and methods: Young ( $\leq 40$ years old) patients with oral SCC were retrospectively enrolled, and each young patient was matched with an old ( $\geq 60$ years old) oral SCC patient. Associations between the NLR and clinicopathological variables were analyzed by the chi-square test, and the Kaplan-Meier method was used to analyze recurrence-free survival (RFS) and disease-specific survival (DSS) rates.

Results: A total of 103 young patients were enrolled, and compared to the old group, the young group had a significantly lower NLR value $(p=0.012)$. In the young group, the 5-year RFS and DSS rates were $82 \%$ and $85 \%$, respectively. In the old group, the 5-year RFS and DSS rates were $65 \%$ and $71 \%$, respectively, and the differences between the groups were significant (both $p<0.05$ ). In the young patients with an NLR $\leq 2.56$, the 5-year DSS rate was $93 \%$, while in the young patients with an NLR $>2.56$, the 5 -year DSS rate was $76 \%$. This difference was significant $(p=0.020)$. A further Cox model analysis confirmed that the NLR was an independent prognostic factor for DSS.

Conclusion: Young patients with oral SCC have a better prognosis than old oral SCC patients, and the NLR is significantly associated with DSS in young patients.

Keywords: oral squamous cell carcinoma, young patient, head and neck cancer, neutrophil-to-lymphocyte ratio, survival analysis

\section{Introduction}

Oral squamous cell carcinoma (SCC) is a common and often lethal form of head and neck SCC; traditionally, it is thought to affect men older than 60 years old after extensive alcohol and tobacco use. However, an alarming increase in the incidence of oral SCC among young patients has been noted by other authors. ${ }^{1,2}$ Generally, young patients are less likely to have a history of alcohol or tobacco exposure, and the common risk factors might act as inflammation promoters that upregulate the neutrophil-to-lymphocyte ratio (NLR). ${ }^{3-7}$ The NLR is a well-known inflammatory marker, several cytokines, and angiogenic factors can be produced by neutrophils, and these agents play important roles in promoting tumor development, and lymphocytes are associated with immune surveillance and act by eliminating cancer cells, a decreased lymphocyte level is related to poorer ability to eliminate cancer cells. Increasingly stronger evidence has indicated the prognostic role of the NLR in traditional head and neck SCC, ${ }^{3-8}$ but no researchers have analyzed whether there is a similar phenomenon in young patients. Moreover, the prognosis in young patients
Correspondence: Kang Gan

Department of Oral Medicine, The First affiliated hospital of Zhengzhou University, Zhengzhou, People's Republic of China

Tel +8637165587239

Fax +8637165587998

Email gangkang@I26.com 
remains controversial, although many comparable outcomes have been reported in young and old patients. A recent study suggested that young patients may actually have worse recurrence rates and a worse prognosis, ${ }^{8}$ which suggests that young oral SCC patients might be different from traditional oral SCC patients. Therefore, the current study evaluated the prognosis of young patients with oral SCC with a focus on the value of the pretreatment NLR.

\section{Patients and methods}

The Zhengzhou University institutional research committee approved our study, all participants provided written informed consent for medical research prior to their initial treatment, this study was conducted in accordance with the Declaration of Helsinki. All methods were performed in accordance with the relevant guidelines and regulations.

Consecutive patients from January 1995 to July 2018 surgically treated for primary oral SCC were retrospectively reviewed. Eligible patients were those who were $\leq 40$ or $\geq 60$ years old, of any sex, able to provide written informed consent, and not previously treated for oral SCC. Data regarding age, sex, TNM stage, the pretreatment NLR, pathological characteristics, surgical treatment, and followup were collected and analyzed. All pathological sections were re-reviewed, and all patients were routinely followed up by out-patient clinic, telephone, email, or we-chat.

In our cancer center, neck dissection was routinely suggested for any stage of oral SCC; in extreme cases, the wait-and-see approach was reserved only for patients with a very small primary lesion. Adjuvant treatments including radiotherapy or chemoradiotherapy were suggested if there was presence of high-risk factors including $\mathrm{T} 3$ to $\mathrm{T} 4$ or N2 to N3 disease, positive surgical margins, extracapsular extension, lymphovascular invasion, and perineural invasion. The regimens of chemotherapy were mainly platinum-based drugs.

Systematic examination was routinely performed with the help of ultrasound, CT, MRI, and/or PET-CT examinations. TNM staging was performed based on the AJCC 7th edition classification.

The NLR was defined as the absolute neutrophil count divided by the absolute lymphocyte count measured within 2 weeks before the initial treatment. ${ }^{3-9}$ The cutoff values calculated from the ROC curve, mean, tertiles, or median in previous studies varied from 1.98 to $5,,^{3-9}$ and the standard cutoff value remains undetermined. In the current study, the cutoff value was defined as the mean value of the NLR based on our previous studies. ${ }^{8,9}$
The patients $\leq 40$ years old were enrolled as a young group. Matched patients $\geq 60$ years old were selected. In this study, each young patient was matched to an old patient, and the patients were matched by sex, smoking status, drinking status, primary site, margin status, disease stage (stage I/II vs stage III/IV), and neck node status. The matched patients $\geq 60$ years old were enrolled as the old group. ${ }^{9,10}$ Drinkers were defined as patients who consumed at least one alcoholic drink per day for at least 1 year, $^{11,12}$ and smokers were defined as patients who smoked on a daily basis. ${ }^{12}$

The chi-square test was used to analyze the associations between the NLR and clinicopathological variables. Recurrence-free survival (RFS) and disease-specific survival (DSS) were calculated from the date of surgery to the date of the event or the last follow-up. The Kaplan-Meier approach was used to calculate the RFS and DSS rates. Factors that were significant in univariate analysis (logrank method) were then analyzed by Cox model analysis to identify the independent risk factors for the RFS and DSS rates. All statistical analyses were performed by using SPSS 20.0, and $p<0.05$ was considered significant.

\section{Results}

A total of 103 (53 female and 50 male) young patients were enrolled, all patients underwent a neck dissection operation, a negative margin was achieved in all patients, and no patients had chronic steroid use or an autoimmune disease. The clinicopathological characteristics of the two groups are presented in Table 1. More patients received adjuvant therapy in young group, including radiotherapy and chemotherapy, than those in the old group $(p<0.001)$, and the NLR value was significantly lower in the young patients than in the old patients $(p=0.012)$. There was no apparent difference regarding adverse pathological characteristics (all $p>0.05$ ).

During our follow-up, which had a mean time of 89.9 (range: 7-205) months, recurrence occurred in 18 and 34 patients in the young and old groups, respectively. In the young group, there were 5 local recurrences, 7 regional recurrences, 3 locoregional recurrences, and 3 distant recurrences, while in the old group, there were 9 local recurrences, 10 regional recurrences, 12 locoregional recurrences, and 3 distant recurrences; the difference between the groups was not significant $(p=0.501$ ). The 5 -year RFS rates in the young and old groups were $82 \%$ and $65 \%$, respectively, and the difference was significant (Figure 1, $p=0.014$ ). Diseaserelated death occurred in 11 and 27 patients in the young 
Table I Clinical pathologic information of the two groups

\begin{tabular}{|c|c|c|c|}
\hline Variables & $\begin{array}{l}\text { Young Group } \\
(n=103)\end{array}$ & $\begin{array}{l}\text { Old group } \\
(n=103)\end{array}$ & $p$ \\
\hline \multicolumn{4}{|l|}{ Sex } \\
\hline Female & 53 & 53 & \\
\hline Male & 50 & 50 & 1.000 \\
\hline \multicolumn{4}{|l|}{ Smoker } \\
\hline Yes & 10 & 10 & \\
\hline No & 93 & 93 & 1.000 \\
\hline \multicolumn{4}{|l|}{ Drinker } \\
\hline Yes & 8 & 8 & \\
\hline No & 95 & 95 & 1.000 \\
\hline \multicolumn{4}{|l|}{ Primary site } \\
\hline Tongue & 53 & 53 & \\
\hline Mouth floor & 20 & 20 & \\
\hline Lower gingiva & 20 & 20 & \\
\hline Buccal & 10 & 10 & 1.000 \\
\hline \multicolumn{4}{|l|}{ Tumor stage } \\
\hline $\mathrm{TI}+\mathrm{T} 2$ & 83 & 83 & \\
\hline $\mathrm{T} 3+\mathrm{T} 4$ & 20 & 20 & 1.000 \\
\hline \multicolumn{4}{|l|}{ Node stage } \\
\hline No & 75 & 75 & \\
\hline $\mathrm{N}+$ & 28 & 28 & 1.000 \\
\hline \multicolumn{4}{|l|}{ Disease stage } \\
\hline $\mathrm{I} / \mathrm{II}$ & 66 & 66 & \\
\hline III/IV & 37 & 37 & 1.000 \\
\hline \multicolumn{4}{|l|}{ Perineural invasion } \\
\hline Yes & 19 & 22 & \\
\hline No & 84 & 81 & 0.601 \\
\hline \multicolumn{4}{|l|}{ Lymphovascular invasion } \\
\hline Yes & 15 & 18 & \\
\hline No & 88 & 85 & 0.569 \\
\hline \multicolumn{4}{|l|}{ Differentiation } \\
\hline Well & 47 & 40 & \\
\hline Moderate & 43 & 52 & \\
\hline Poor & 13 & II & 0.453 \\
\hline NLR (mean) & 2.56 & 2.81 & 0.012 \\
\hline \multicolumn{4}{|l|}{ Treatment } \\
\hline$S$ & 6 & 26 & \\
\hline$S+R$ & 86 & 72 & \\
\hline$S+R+C$ & II & 5 & $<0.001$ \\
\hline
\end{tabular}

Abbreviations: S, surgery; R, radiotherapy; C, chemotherapy; NLR, neutrophil-tolymphocyte ratio; NS, not significant.

and old groups, respectively. The 5-year DSS rates in the young and old groups were $85 \%$ and $71 \%$, respectively, and the difference was significant (Figure 2, $p=0.010$ ).

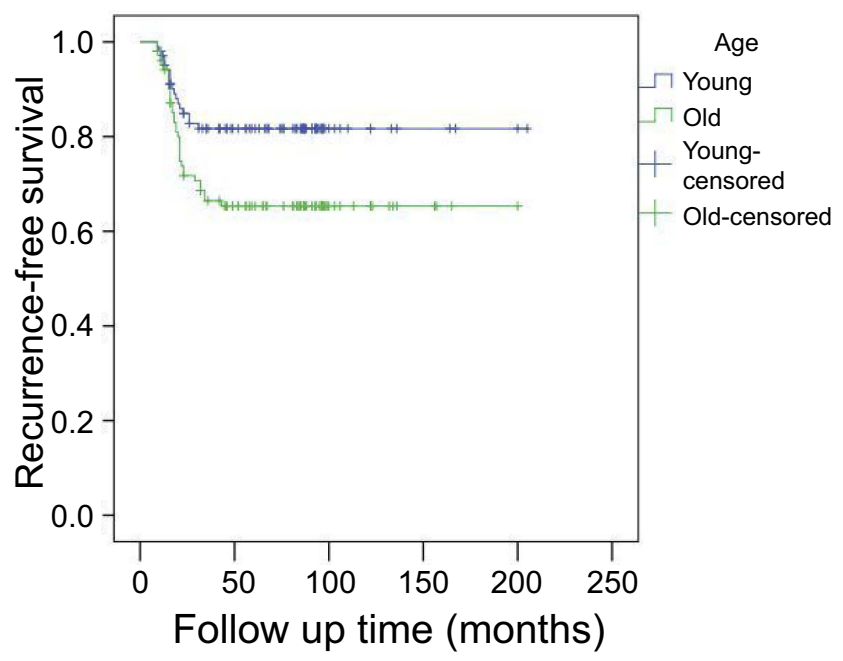

Figure I Comparison of recurrence-free survival between young and old groups $(p=0.014)$.

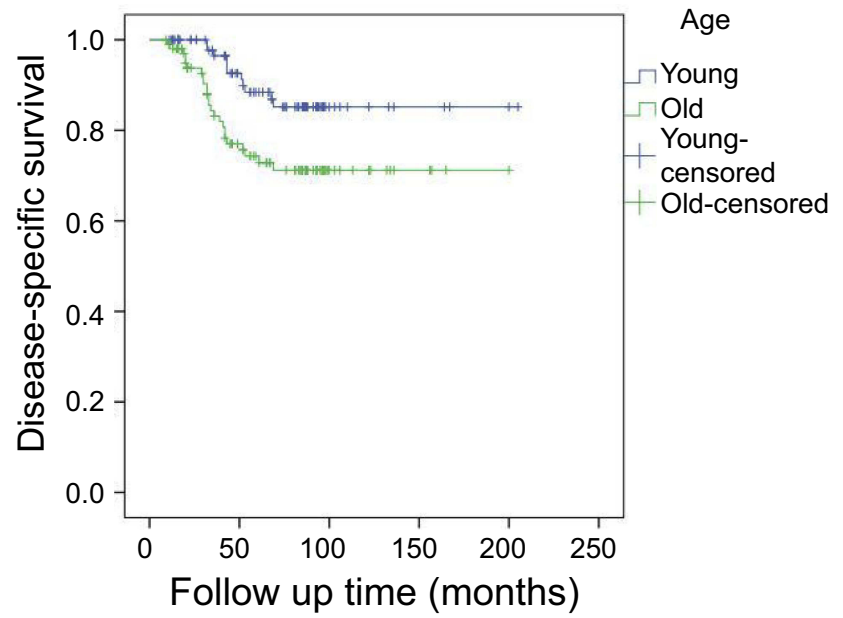

Figure 2 Comparison of disease-specific survival between young and old groups $(p=0.010)$.

The associations between the NLR and clinicopathological variables are presented in Table 2. A high NLR was significantly related to advanced-stage disease and poor differentiation. There were no positive associations between the NLR and sex, tumor stage, node stage, perineural invasion, or lymphovascular invasion.

Univariate analysis (log-rank analysis) of RFS in the young group showed that in the patients with an $\mathrm{NLR} \leq 2.56$, the 5-year RFS rate was $83 \%$, while in the patients with an NLR $>2.56$, the 5-year RFS rate was $80 \%$, and the difference was not significant $(p=0.643)$. Other factors including the primary site, disease stage, tumor differentiation, radiotherapy use, and lymphovascular invasion status were significantly associated with RFS 
Table 2 Association between neutrophil-to-lymphocyte ratio (NLR) and clinical pathologic characteristics in young patients with oral squamous cell carcinoma

\begin{tabular}{|c|c|c|c|}
\hline \multirow[t]{2}{*}{ Variables } & \multicolumn{2}{|l|}{ NLR } & \multirow[t]{2}{*}{$P$} \\
\hline & $\begin{array}{l}\leq 2.56 \\
(n=55)\end{array}$ & $\begin{array}{l}>2.56 \\
(n=48)\end{array}$ & \\
\hline \multicolumn{4}{|l|}{ Sex } \\
\hline Female & 28 & 25 & \\
\hline Male & 27 & 23 & 0.905 \\
\hline \multicolumn{4}{|l|}{ Smoker } \\
\hline Yes & 3 & 7 & \\
\hline No & 52 & 41 & 0.182 \\
\hline \multicolumn{4}{|l|}{ Drinker } \\
\hline Yes & 2 & 6 & \\
\hline No & 53 & 42 & 0.141 \\
\hline \multicolumn{4}{|l|}{ Tumor stage } \\
\hline $\mathrm{TI}+\mathrm{T} 2$ & 48 & 35 & \\
\hline $\mathrm{T} 3+\mathrm{T} 4$ & 7 & 13 & 0.066 \\
\hline \multicolumn{4}{|l|}{ Node stage } \\
\hline No & 44 & 31 & \\
\hline $\mathrm{N}+$ & 11 & 17 & 0.079 \\
\hline \multicolumn{4}{|l|}{ Disease stage } \\
\hline $\mathrm{I} / \mathrm{II}$ & 45 & 21 & \\
\hline III/IV & 10 & 27 & $<0.001$ \\
\hline \multicolumn{4}{|l|}{ Perineural invasion } \\
\hline Yes & 7 & 12 & \\
\hline No & 48 & 36 & 0.109 \\
\hline \multicolumn{4}{|l|}{ Lymphovascular invasion } \\
\hline Yes & 5 & 10 & \\
\hline No & 50 & 38 & 0.092 \\
\hline \multicolumn{4}{|l|}{ Differentiation } \\
\hline Well & 32 & 15 & \\
\hline Moderate & 18 & 25 & \\
\hline Poor & 5 & 8 & 0.023 \\
\hline
\end{tabular}

Abbreviation: NS, not significant.

(all $p<0.05$ ). In a further Cox model analysis, the primary site, disease stage, and lymphovascular invasion status were independent predictors of RFS (Table 3).

Univariate analysis (log-rank analysis) of DSS in the young group showed that in the patients with an $\mathrm{NLR} \leq 2.56$, the 5 -year DSS rate was $93 \%$, while in the patients with an NLR $>2.56$, the 5-year DSS rate was $76 \%$, and the difference was significant (Figure 3, $p=0.020$ ). Other factors including the primary site, disease stage, tumor differentiation, perineural invasion status, and radiotherapy use were also significantly associated with DSS (all $p<0.05$ ). In a further Cox model analysis, a high NLR was also an independent predictor of worse DSS (Table 4).

\section{Discussion}

Oral SCC is uncommon in patients less than 40 years old, and no overall consensus concerning the difference in prognosis between old and young patients has been reached. One of the main findings in the current study was that the prognosis of young patients was significantly better than that of old patients, and this finding conflicted some with the findings of previous reports. Farquhar et al, ${ }^{13}$ found that although there was no difference in overall mortality, young patients were more likely to recur within 3 years than old patients, and the authors concluded that oral SCC among young patients may be distinct from traditional oral SCC. Hilly et al, ${ }^{14}$ described recurrence in similar proportions of patients in young and old groups (38\% and $29.9 \%$, respectively), and a Kaplan-Meier analysis yielded no between-group differences in disease-free or overall survival. Pitman et al, ${ }^{15}$ noted that the 3 -year disease-free survival rate of a group of patients older than 40 years old was $55.0 \%$, while the 3 -year disease-free survival rate was $53.3 \%$ in young groups, and the authors suggested that the outcomes of treatment for tongue SCC in young patients were similar to those in patients older than 40 with a similar extent of disease. Friedlander et al, ${ }^{16}$ reported that younger patients with tongue SCC had a higher rate of locoregional recurrence than older patients, but this difference did not translate into a survival difference. However, the results of these reports were potentially influenced by one or more confounding factors that affected the interpretation of the outcomes. First, the definition of young patients was inconsistent, and the cutoff age varied from 30 years to 40 years. Second, the factors of smoking and alcohol use were never matched to prevent interference in previous studies; ${ }^{13-16}$ the incidences of smokers and drinkers were usually higher in the older groups, and alcohol use and smoking have immunosuppressive effects and are associated with a worse prognosis, ${ }^{17,18}$ which would lead to decreased disease control in the older, control groups. In the current study, as many confounding factors as possible were matched for increasing the study reliability, but there were still some differences regarding the baseline data of the two groups, which might partially explain the prognosis variation. Owing to the better toleration of side effects, more adjuvant treatments were performed in the young patients, and this increased treatment surely could improve patient prognosis. On the other hand, it was interesting to find that 
Table 3 Predictors for recurrence-free survival in young patients with oral squamous cell carcinoma

\begin{tabular}{|c|c|c|c|}
\hline \multirow[t]{2}{*}{ Variables } & \multirow{2}{*}{$\begin{array}{l}\text { Log-rank analysis } \\
P\end{array}$} & \multicolumn{2}{|l|}{ Cox model } \\
\hline & & HR(95\% Cl) & $p$ \\
\hline Sex & 0.435 & & \\
\hline Smoker & 0.223 & & \\
\hline Drinker & 0.533 & & \\
\hline Primary site (tongue vs other sites) & 0.009 & $2.497(1.562-7.246)$ & $<0.001$ \\
\hline Tumor stage $(\mathrm{TI}+\mathrm{T} 2$ vs $\mathrm{T} 3+\mathrm{T} 4)$ & 0.064 & & \\
\hline Node stage (N0 vs $\mathrm{N}+$ ) & 0.111 & & \\
\hline Disease stage (I/II vs III/IV) & 0.008 & $3.458(1.597-9.335)$ & $<0.001$ \\
\hline Perineural invasion & 0.099 & & \\
\hline Lymphovascular invasion & 0.006 & $2.002(1.346-4.886)$ & 0.003 \\
\hline Differentiation (Well vs Moderate + Poor) & 0.018 & $2.946(0.943-6.669)$ & 0.099 \\
\hline $\operatorname{NLR}(\leq 2.56$ vs $>2.56)$ & 0.643 & & \\
\hline Radiotherapy & 0.033 & $0.632(0.421-1.165)$ & 0.154 \\
\hline Chemotherapy & 0.875 & & \\
\hline
\end{tabular}

Abbreviation: NLR, neutrophil-to-lymphocyte ratio.

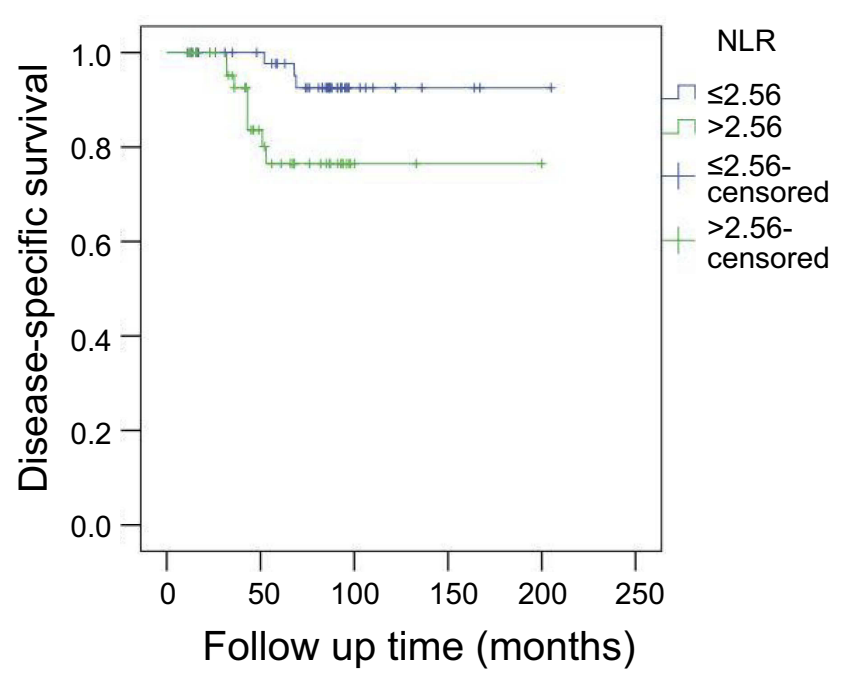

Figure 3 Disease-specific survival in patients with different neutrophil-to-lymphocyte ratio (NLR) $(p=0.020)$.

the NLR value was significantly lower in the young patients than in the old patients, and previous strong evidence has shown that a high NLR value is a predictor of a worse prognosis in head and neck SCC. ${ }^{3-7}$ Additionally, although we did not have the HPV status of the patients, because of the higher sexual activity in young patients, there might be higher rate of HPV infection in young patients, and several authors had shown the correlation between HPV + and better prognosis in young people. ${ }^{19,20}$ Furthermore, other unassessed factors, including poor oral hygiene and poor dentures in the old patients, might also be responsible for this finding.
The significance of the NLR in traditional head and neck SCC has been widely analyzed. Yu et al, ${ }^{21}$ described that head and neck cancer patients with an elevated pretreatment NLR in the peripheral blood were prone to local invasion and distant recurrence and had a poor prognosis. Kano et al, ${ }^{22}$ analyzed data from 285 patients with head and neck cancer treated with concurrent chemotherapy and found that there were significant relationships between a high NLR and hypopharyngeal or oropharyngeal cancer, $\mathrm{N} 2 \mathrm{~b}$ to N3 stages, T3 to T4 stages, and clinical stages III to IV and that in further survival assessments, a high NLR was significantly associated with decreases in disease-free survival and overall survival. However, whether there are similar findings in young oral SCC patients remains unknown. The current study is the first to report that a high NLR is related to adverse pathological characteristics and DSS but not RFS.

The exact mechanisms underlying the association between the NLR and the prognosis remain unknown, but some possible explanations can be inferred from previous evidence. On the one hand, an elevated neutrophil level is a sign of local and systemic inflammatory responses. Several cytokines and angiogenic factors can be produced by neutrophils, and these agents play important roles in promoting tumor development. ${ }^{23}$ These hematological markers are also surrogate markers of cancer cachexia, which is related to poor survival. ${ }^{24,25}$ On the other hand, lymphocytes are associated with immune surveillance and act by eliminating cancer cells, ${ }^{26}$ a decreased lymphocyte level is related to poorer ability to eliminate 
Table 4 Predictors for disease-specific survival in young patients with oral squamous cell carcinoma

\begin{tabular}{|c|c|c|c|}
\hline \multirow[t]{2}{*}{ Variables } & \multirow{2}{*}{$\begin{array}{l}\text { Log-rank analysis } \\
p\end{array}$} & \multicolumn{2}{|l|}{ Cox model } \\
\hline & & HR(95\% Cl) & $p$ \\
\hline Sex & 0.641 & & \\
\hline Smoker & 0.115 & & \\
\hline Drinker & 0.068 & & \\
\hline Primary site (tongue vs other sites) & 0.007 & $2.047(1.687-5.227)$ & 0.004 \\
\hline Tumor stage $(\mathrm{TI}+\mathrm{T} 2$ vs $\mathrm{T} 3+\mathrm{T} 4)$ & 0.245 & & \\
\hline Node stage (N0 vs $\mathrm{N}+$ ) & 0.087 & & \\
\hline Disease stage (I/II vs III/IV) & 0.001 & $4.281(2.101-10.223)$ & $<0.001$ \\
\hline Perineural invasion & 0.015 & $2.110(1.347-4.613)$ & 0.009 \\
\hline Lymphovascular invasion & 0.110 & & \\
\hline Differentiation (Well vs Moderate + Poor) & 0.003 & $2.348(1.612-6.759)$ & 0.003 \\
\hline NLR $(\leq 2.56$ vs $>2.56)$ & 0.020 & $1.874(1.075-2.886)$ & 0.037 \\
\hline Radiotherapy & 0.031 & $0.845(0.43 I-1.22 I)$ & 0.098 \\
\hline Chemotherapy & $0.4 \mathrm{II}$ & & \\
\hline
\end{tabular}

Abbreviation: NLR, neutrophil-to-lymphocyte ratio.

cancer cells. Therefore, a high NLR is considered to predict a worse prognosis.

We failed to note there is a positive relationship between NLR and the RFS in young patients, the finding was conflicted with previous researchers, ${ }^{9,21,22}$ possible explanation was our different subjects, only young patients were enrolled in the current study, young oral SCC patients might be a distinct entity from traditional oral SCC patients owing to different pathogenic factors, additionally, as our result described, lower NLR in young patients might mean relatively milder negative effect than higher NLR in old patients.

The limitations of the current study must be acknowledged. First, the number of events in the younger cohort was relatively small, which might decrease the statistical power of our research. A study with a larger sample size is needed to clarify some questions. Second, this is a retrospective study, and there is an inherent bias that might decease the statistical power. Third, it should be recognized that neutrophil and lymphocyte counts are nonspecific parameters because they can be influenced by concomitant conditions, such as infections or inflammation.

In summary, young patients with oral SCC have a better prognosis than old patients, and the NLR is significantly associated with DSS in young patients.

\section{Availability of data and materials}

All data generated or analyzed during this study are included in this published article. The primary data can be obtained from the corresponding author.

\section{Ethics Statement}

The Zhengzhou University institutional research committee approved our study, all participants provided written informed consent for medical research prior to their initial treatment, this study was conducted in accordance with the Declaration of Helsinki. All methods were performed in accordance with the relevant guidelines and regulations. All the material came from our cancer center, and the consent to publish was obtained from all patients.

\section{Author contributions}

All authors contributed to data analysis, drafting or revising the article, gave final approval of the version to be published, and agree to be accountable for all aspects of the work.

\section{Disclosure}

The authors report no conflicts of interest in this work.

\section{References}

1. Ng JH, Iyer NG, Tan M-H, Edgren G. Changing epidemiology of oral squamous cell carcinoma of the tongue: a global study. Head Neck. 2017;39:297-304. doi:10.1002/hed.24589

2. Patel SC, Carpenter WR, Tyree S, et al. Increasing incidence of oral tongue squamous cell carcinoma in young white women, age 18 to 44 years. $J$ Clin Oncol. 2011;29:1488-1494. doi:10.1200/JCO.2010.31.7883

3. Chen MF, Tsai MS, Chen WC, Chen PT. Predictive value of the pretreatment neutrophil-to-lymphocyte ratio in head and neck squamous cell carcinoma. J Clin Med. 2018;20:7.

4. Mascarella MA, Mannard E, Silva SD, Zeitouni A. Neutrophil-tolymphocyte ratio in head and neck cancer prognosis: a systematic review and meta-analysis. Head Neck. 2018;40:1091-1100. doi:10.1002/hed.25075 
5. Rosculet N, Zhou XC, Ha P, et al. Neutrophil-to-lymphocyte ratio: prognostic indicator for head and neck squamous cell carcinoma. Head Neck. 2017;39:662-667. doi:10.1002/hed.24658

6. Sumner WA, Stokes WA, Oweida A, et al. Survival impact of pretreatment neutrophils on oropharyngeal and laryngeal cancer patients undergoing definitive radiotherapy. J Transl Med. 2017;15:168. doi:10.1186/s12967-017-1268-7

7. Valero C, Pardo L, López M, et al. Pretreatment count of peripheral neutrophils, monocytes, and lymphocytes as independent prognostic factor in patients with head and neck cancer. Head Neck. 2017;39:219-226. doi:10.1002/hed.24561

8. Fang Q, Liu F, Seng D. Oncologic outcome of parotid mucoepidermoid carcinoma in pediatric patients. Cancer Manag Res. 2019;11:1081-1085. doi:10.2147/CMAR.S192788

9. Fang Q, Li P, Qi J, Luo R, Chen D, Zhang X. Value of lingual lymph node metastasis in patients with squamous cell carcinoma of the tongue. Laryngoscope. 2019. doi:10.1002/lary.27927

10. Blanchard P, Belkhir F, Temam S, et al. Outcomes and prognostic factors for squamous cell carcinoma of the oral tongue in young adults: a singleinstitution case-matched analysis. Eur Arch Otorhinolaryngol. 2017;274:1683-1690. doi:10.1007/s00405-016-4419-1

11. Fang QG, Shi S, Liu FY, Sun CF. Squamous cell carcinoma of the oral cavity in ever smokers: a matched-pair analysis of survival. J Craniofac Surg. 2014;25:934-937. doi:10.1097/SCS.0000000000000710

12. Bachar G, Hod R, Goldstein DP, et al. Outcome of oral tongue squamous cell carcinoma in patients with and without known risk factors. Oral Oncol. 2011;47:45-50. doi:10.1016/j.oraloncology.2010.11.003

13. Farquhar DR, Tanner AM, Masood MM, et al. Oral tongue carcinoma among young patients: an analysis of risk factors and survival. Oral Oncol. 2018;84:7-11. doi:10.1016/j.oraloncology.2018.06.014

14. Hilly O, Shkedy Y, Hod R, et al. Carcinoma of the oral tongue in patients younger than 30 years: comparison with patients older than 60 years. Oral Oncol. 2013;49:987-990. doi:10.1016/j. oraloncology.2013.07.005

15. Pitman KT, Johnson JT, Wagner RL, Myers EN. Cancer of the tongue in patients less than forty. Head Neck. 2000;22:297-302.

16. Friedlander PL, Schantz SP, Shaha AR, Yu G, Shah JP. Squamous cell carcinoma of the tongue in young patients: a matched-pair analysis. Head Neck. 1998;20:363-368.
17. Gerson SJ. Oral cancer. Crit Rev Oral Biol Med. 1990;1:153-166. doi:10.1177/10454411900010030101

18. Chen AM, Chen LM, Vaughan A, et al. Head and neck cancer among lifelong never-smokers and ever-smokers: matched-pair analysis of outcomes after radiation therapy. Am J Clin Oncol. 2011;34:270-275. doi:10.1097/COC.0b013e3181dea40b

19. Wang F, Zhang H, Xue Y, et al. A systematic investigation of the association between HPV and the clinicopathological parameters and prognosis of oral and oropharyngeal squamous cell carcinomas. Cancer Med. 2017;6:910-917. doi:10.1002/cam4.1045

20. Abbate V, Dell'Aversana Orabona G, Salzano G, et al. Pre-treatment Neutrophil-to-Lymphocyte Ratio as a predictor for occult cervical metastasis in early stage (T1-T2 cN0) squamous cell carcinoma of the oral tongue. Surg Oncol. 2018;27:503-507. doi:10.1016/j. suronc.2018.06.002

21. Yu Y, Wang H, Yan A, et al. Pretreatment neutrophil to lymphocyte ratio in determining the prognosis of head and neck cancer: a metaanalysis. BMC Cancer. 2018;18:383. doi:10.1186/s12885-0184242-8

22. Kano S, Homma A, Hatakeyama H, et al. Pretreatment lymphocyteto-monocyte ratio as an independent prognostic factor for head and neck cancer. Head Neck. 2017;39:247-253. doi:10.1002/hed.24576

23. Tecchio C, Scapini P, Pizzolo G, Cassatella MA. On the cytokines produced by human neutrophils in tumors. Semin Cancer Biol. 2013;23:159-170. doi:10.1016/j.semcancer.2013.02.004

24. Kawakita D, Tada Y, Imanishi Y, et al. Impact of hematological inflammatory markers on clinical outcome in patients with salivary duct carcinoma: a multi-institutional study in Japan. Oncotarget. 2017;8:1083-1091. doi:10.18632/oncotarget.13565

25. Liu F, Cheng G, Fang Q, Sun Q. Natural history of untreated squamous cell carcinoma of the head and neck. Clin Otolaryngol. 2018; Epub ahead of print. doi:10.1111/coa.13260.

26. Mohammed ZM, Going JJ, Edwards J, Elsberger B, Doughty JC, McMillan DC. The relationship between components of tumour inflammatory cell infiltrate and clinicopathological factors and survival in patients with primary operable invasive ductal breast cancer. $\mathrm{Br}$ J Cancer. 2012;107:864-873. doi:10.1038/bjc.2012.347

\section{Publish your work in this journal}

Cancer Management and Research is an international, peer-reviewed open access journal focusing on cancer research and the optimal use of preventative and integrated treatment interventions to achieve improved outcomes, enhanced survival and quality of life for the cancer patient
The manuscript management system is completely online and includes a very quick and fair peer-review system, which is all easy to use. Visit http://www.dovepress.com/testimonials.php to read real quotes from published authors. 